\title{
Special issue on theory and application of network algorithms in biology
}

\author{
Karthik Raman ${ }^{1,2,3} \cdot$ Ananth Kalyanaraman ${ }^{4}$
}

Published online: 1 July 2019

(C) Indian Institute of Technology Madras 2019

\section{Overview}

The proliferation of high-throughput technologies coupled with a surging interest in understanding the structure and dynamic behaviour of naturally evolved and engineered systems has resulted in a vast availability of data of enormous scale and unprecedented complexity. This is exemplified in the life sciences, where high-throughput technologies have enabled scientists to observe a biological system at all scales, from the molecular to the cellular to the population levels. However, such raw data are often not directly informative; they need to be digitally transformed and analysed, before which useful information can be extracted about the underlying system.

A key characteristic of such data is the inherent interconnectivity among the entities captured by the data-e.g. a set of protein molecules interacting to form a protein interaction or signalling network; or a set of bacterial cells

Karthik Raman

kraman@iitm.ac.in

Ananth Kalyanaraman

ananth@wsu.edu

1 Department of Biotechnology, Indian Institute of Technology Madras, Chennai 600 036, India

2 Initiative for Biological Systems Engineering, IIT Madras, Chennai, India

3 Robert Bosch Centre for Data Science and Artificial Intelligence (RBC-DSAI), IIT Madras, Chennai, India

4 School of Electrical Engineering and Computer Science, Washington State University, Pullman, WA, USA signalling each other to form an antibiotic-resistant biofilm; or a set of infectious pathogens spreading across a wide population of hosts and vectors in a city. Consequently, graph-theoretic/network representations and analytical methods have taken centre stage in the modelling and understanding of the behaviour of biological systems at scale.

The goal of this special issue is to highlight state-of-theart research in network science algorithms and applications stemming from the biological sciences. Network biology and related network science topics represent a broad and vibrant research area. While it is not possible to cover the entire breadth of research activities being actively pursued in this area in one special issue, this special issue showcases five peer-reviewed papers covering a wide variety of topics in network biology-ranging from methods for the analysis of biomolecular networks (transcriptomic and genome-wide) to network-based representation and modelling of dynamics in population-scale data. All these papers were received in response to a widely publicised open call for papers and underwent a thorough peer-review process prior to their selection.

More specifically, Gadiyaram et al. present a perturbation-based approach to identify important nodes (elements) of a network and evaluate their approach in the context of protein structure networks. Kumar and Vinod present a temporal model to study the trajectory of single-cell transcriptomic data obtained from pancreatic islets in healthy and type 2 diabetic tissues. Ravichandran and Chandra present a systematic comparison of knowledge-based and statistical methods for analysing genome-wide networks obtained using various omics technologies. 
At the population scale, Sinha et al. present an evolutionary game-theoretic approach to model the interactions of individuals in a population and to consequently obtain insights into the structure and behaviour of such populations. Adiga et al. present a review of graphical dynamic systems and their applications to various biosocial systems including (but not limited to) mammalian immune systems, epidemiological networks, gene interaction networks and spatial ecology networks.

Network modelling and algorithms have a critical role to play in the advancement of our understanding and characterisation of biological systems, with their ability to convert raw data into information and subsequently into actionable insights and knowledge. We hope that these articles will collectively provide a window into the diversity of research topics actively pursued in the area of biological network science, alongside the related challenges and opportunities, and will further motivate a curious reader to explore new research venues for investigation and contribution.

Publisher's Note Springer Nature remains neutral with regard to jurisdictional claims in published maps and institutional affiliations. 